\title{
Nódulo de la hermana Mary Joseph: una manifestación inicial poco usual de adenocarcinoma de páncreas
}

\section{Sister Mary Joseph nodule: an unusual initial manifestation of pancreas adenocarcinoma}

Correspondencia Martin Chapoñan-Relaiza mchaponan38@gmail.com

Recibido: 20/06/2017 Arbitrado por pares Aprobado: 23/08/2017

Citar como: Chapoñan-Relaiza M, Catacora Cama J, Delgado Gonzales V. Nódulo de la hermana Mary Joseph: una manifestación inicial poco usual de adenocarcinoma de páncreas. Acta Med Peru. 2017;34(4):323-7
Martin Chapoñan-Relaiza ${ }^{1,2}$, José Catacora Cama1 ${ }^{1}$ Victor Delgado Gonzales ${ }^{3}$

1 Servicio de Dermatología, Hospital Nacional Guillermo Almenara Irigoyen. Lima, Perú.

2 Facultad de Medicina, Universidad Nacional Mayor de San Marcos, Lima, Perú.

3 Servicio de Anatomía Patológica, Hospital Nacional Guillermo Almenara Irigoyen. Lima, Perú.

\section{RESUMEN}

Las metástasis cutáneas representan el $10 \%$ de todas las metástasis. El nódulo de la hermana Mary Joseph es una lesión metastásica cutánea umbilical asociada a tumor intraabdominal, que con mayor frecuencia es el carcinoma gástrico. Las metástasis cutáneas de cáncer de páncreas son poco habituales y su localización más frecuente es el ombligo. La mayoría de referencias de la literatura consisten en reportes o series de caso pequeñas. Se reporta el caso de un paciente con lesión metastásica umbilical, nódulo de la hermana Mary Joseph, primaria de adenocarcinoma pancreático, debido a su inusual presentación como manifestación clínica previa al diagnóstico de la neoplasia de origen.

Palabras clave: Nódulo de la hermana María José; Metástasis de la neoplasia; Piel; Páncreas (fuente: DeCS BIREME).

\section{ABSTRACT}

Cutaneous metastases represent $10 \%$ of all metastatic manifestations. Sister Mary Joseph nodule is a cutaneous umbilical metastatic lesion associated to intraabdominal tumors (most frequently gastric carcinoma). Cutaneous metastases from pancreatic cancer are quite uncommon, and their most frequent location is the umbilicus. Most literature references consist in case reports or small case series. We report the case of a patient with an umbilical metastatic lesion (Sister Mary Joseph nodule) from a pancreatic adenocarcinoma, being this an unusual presentation as a clinical manifestation prior to diagnosing the original malignancy.

Keywords: Sister Mary Joseph's nodule; Neoplasm metastasis; Skin; Pancreas (source: MeSH NLM). 


\section{INTRODUCCIÓN}

El nódulo de la hermana Mary Joseph es una lesión cutánea de origen metastásico secundario a una neoplasia de cualquier órgano generalmente intrabdominal. Descrito por primera vez por la hermana Mary Joseph Dempsey, asistente de enfermería del Saint Mary's Hospital (luego Clínica Mayo), y usado por primera vez por William Mayo en la Sociedad de Cirugía de Cincinnati en $1929^{[1]}$. En ocasiones, no se conoce frecuencia exacta, puede ser el único signo de la existencia de una neoplasia, así como el de una posible recurrencia.

La frecuencia de esta manifestación cutánea no está bien definida; sin embargo, en algunos estudios de revisión se estima más frecuente en sexo femenino. Se presentan en aproximadamente 1 a 3\% de las neoplasias intrabdominales, principalmente las del tracto gastrointestinal (Tabla 1). El origen más frecuente es el cáncer gástrico, se le atribuyen al menos el $55 \%$ de los casos, seguido del cáncer colorrectal. El siguiente grupo en frecuencia son las neoplasias genitourinarias (se estima que al menos $10 \%$ son producidas por el cáncer de ovario). Alrededor de $15 \%$ de neoplasias no tiene origen conocido. No existen datos epidemiológicos de nódulo de la hermana Mary Joseph en la literatura nacional ${ }^{[1-8]}$

El objetivo de nuestro estudio es reportar la presencia del nódulo de la hermana Mary Joseph en un paciente con cáncer de páncreas, debido a su presentación inicial previa a los síntomas de la neoplasia, con el fin de realizar un reconocimiento temprano y un manejo oportuno del paciente.

\section{REPORTE DE CASO}

Varón de 47 años, natural y procedente de Huánuco, Perú, quien presentaba desde hace cinco meses una masa umbilical sólida, indolora, que drenaba ocasionalmente material serosanguinolento, con crecimiento lentamente progresivo; luego de un mes, refiere la presencia de dolor abdominal tipo cólico acompañado de náuseas, vómitos esporádicos y baja progresiva de peso.

Es referido al servicio de Medicina Interna del Hospital Nacional Guillermo Almenara (Lima, Perú), quienes realizan exámenes auxiliares y solicitan interconsulta a servicio de Dermatología.

Al examen físico, se observó que el paciente tenía un regular estado general y de nutrición, con signos vitales dentro de rangos normales. En abdomen, se observó la presencia de un nódulo umbilical, eritematoso, de $2,5 \mathrm{~cm}$ de diámetro, con ulceración central, escasa secreción serohemática, de consistencia firme, adherido a planos profundos, no doloroso a la palpación. En el resto del examen se observó ictericia, palidez, distensión abdominal asociada hepatoesplenomegalia, con dolor a la palpación abdominal profunda (Figura 1).

El barrido tomográfico mostraba una masa hipodensa de bordes irregulares en cuerpo y cola de páncreas, múltiples imágenes nodulares hipodensas en parénquima hepático, adenopatías a nivel pericelíaco y peripancreático (Figura 2A); asimismo, se observó una masa hiperdensa, que captaba contraste, de bordes definidos a nivel umbilical (Figura 2B).

Se realizaron biopsias de piel e hígado. La biopsia de piel mostraba un infiltrado a nivel de la dermis compuesto por estructuras glandulares mal definidas moderadamente diferenciadas. El estudio inmunohistoquímico fue positivo para panqueratina y CK7 y negativo para CEA y citoqueratina. El diagnóstico anatomopatológico confirmó la presencia de adenocarcinoma metastásico primario de páncreas (Figura 3). La biopsia hepática mostró tejido hepático infiltrado por adenocarcinoma compatible con metástasis de primario pancreático.

El diagnóstico final fue adenocarcinoma de páncreas con metástasis hepática y cutánea. Se derivó al paciente al servicio de Oncología donde se realizó tratamiento quimioterápico
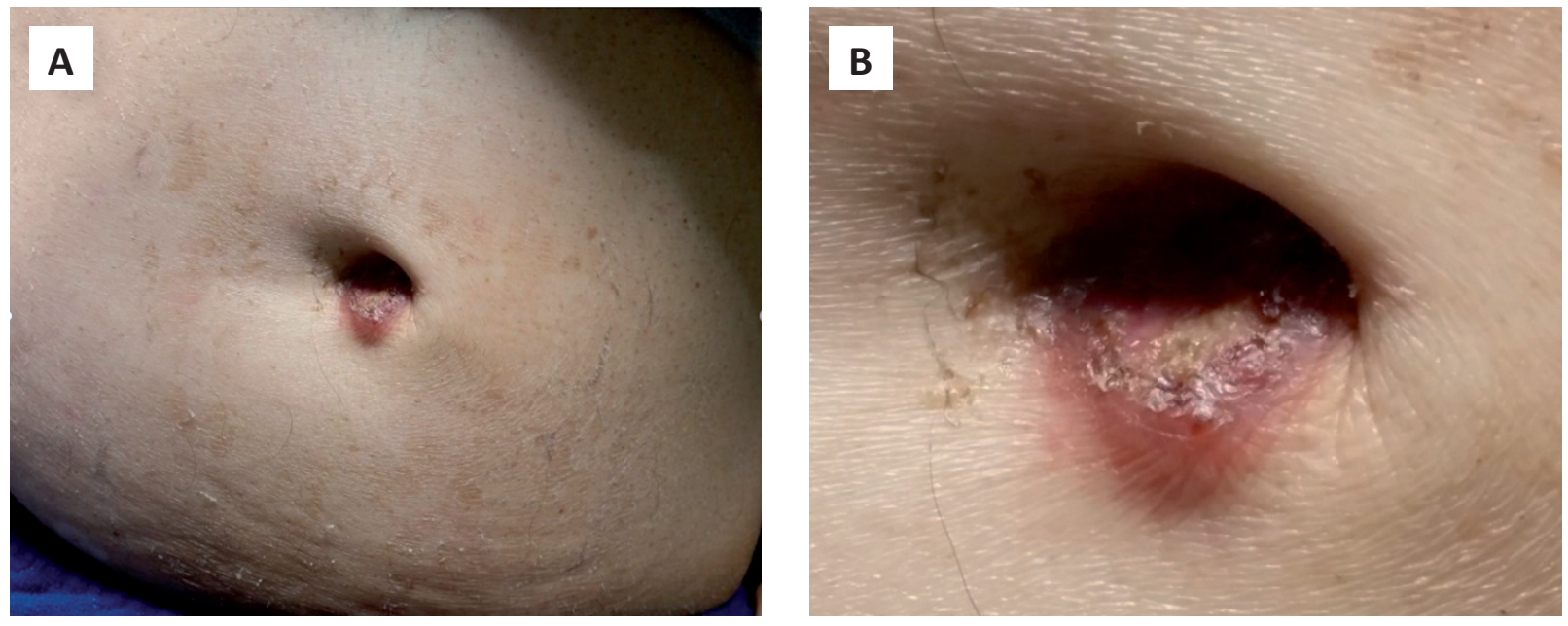

Figura 1. A) Paciente con distensión abdominal y hepatomegalia donde se aprecia lesión nodular en ombligo. B) A mayor aumento, nódulo umbilical de $2,5 \mathrm{~cm}$ de diámetro, de bordes mal definidos, eritematoso, centro ulcerado, no doloroso a la palpación. 

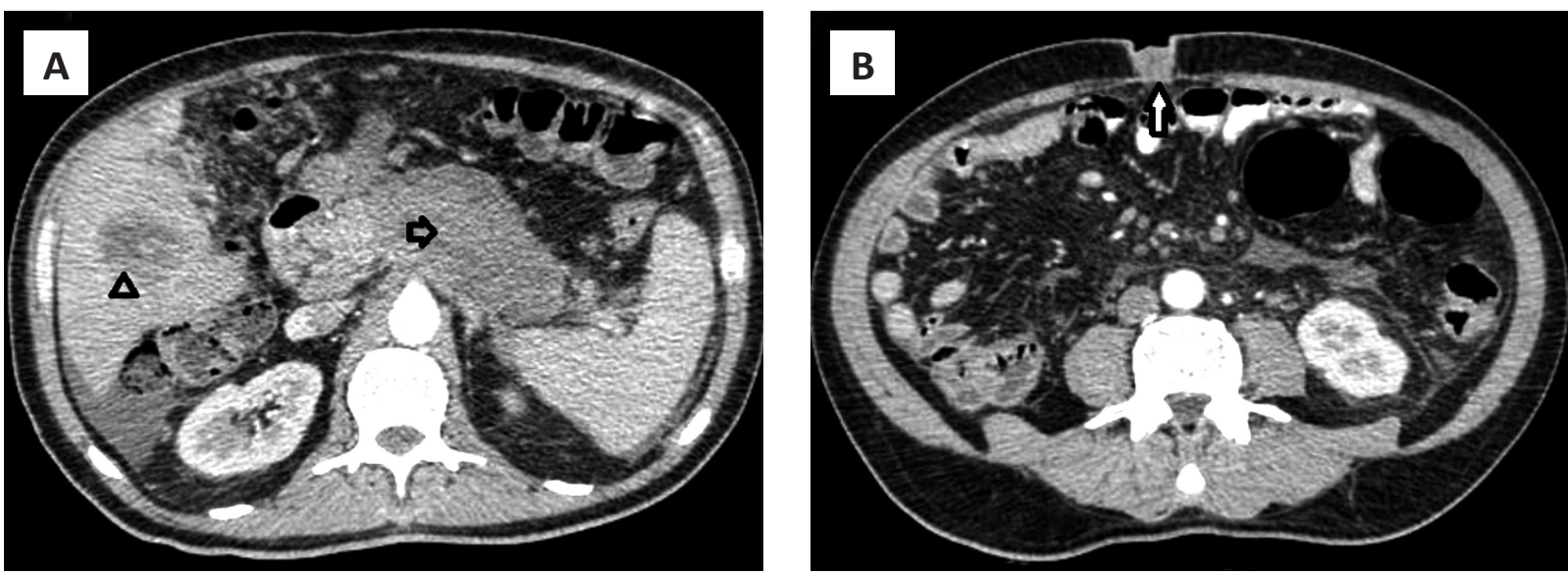

Figura 2. A) Masa pancreática irregular localizada en cuerpo y cola de páncreas (flecha gris) asociada con una masa hipodensa en parénquima hepático compatible con lesión metastásica (cabeza de flecha). B) Lesión isodensa en región umbilical, circunscrita, bordes definidos, compatible con metástasis cutánea de adenocarcinoma de páncreas (flecha blanca).

con Oxiplatino, Irinotecan y 5-fluorouracilo, los cuales se administraron vía endovenosa; no obstante, falleció luego de seis meses de realizado el diagnóstico de la neoplasia primaria.

\section{DISCUSIÓN}

El ombligo raramente es el sitio de origen de un tumor maligno; sin embargo, cuando lo es, el de tipo epidermoide resulta el más frecuente de los tumores umbilicales primarios ${ }^{[2]}$

Los lugares de origen más común del nódulo de Mary Joseph son el estómago y el colon en los hombres y los ovarios en las mujeres. Otras localizaciones incluyen el ciego, intestino delgado, vejiga, trompa uterina, cérvix, próstata, endometrio y páncreas, como el caso del paciente descrito ${ }^{[3,4]}$. En nuestro caso la neoplasia originaria de la lesión metastásica fue el adenocarcinoma de páncreas. Esto lo ubica dentro del grupo de tumores abdominales, pero siendo el páncreas uno de las neoplasias de este grupo que originar el nódulo de la hermana Mary Joseph hace de este caso una presentación menos habitual, así como su forma de inicio como se discute más adelante.

Hasta un $40 \%$ de los casos son expresion de neoplasias recidivantes ${ }^{[5]}$; sin embargo, hay algunos casos (como el de nuestro paciente) de presentación inicial de compromiso cutáneo antes del diagnóstico de la neoplasia primaria.

En la mayoría de los casos de nódulo de María José hay síntomas sugestivos, mientras que en el $15 \%$ de los casos puede ser el primer y único, como en el caso que presentamos ${ }^{[6]}$.

Clínicamente es un nódulo umbilical firme, el cual puede ulcerarse o fisurarse (como en nuestro paciente), puede tener coloración eritematosa, marrón o azulada, a veces pruriginoso, no doloroso a la palpación. Los pacientes suelen tener síntomas gastrointestinales como dolor abdominal, pérdida de peso, distensión abdominal, náuseas ${ }^{[7]}$. La edad de presentación en nuestro paciente fue menor a lo descrito, puesto que el promedio de aparición está alrededor de los 60 años de edad ${ }^{[8]}$.

Otras causas de nódulo umbilical de índole benigno son los papilomas fibroepiteliales, dermatofibromas, malformaciones del conducto onfalomesentérico, cicatrices hipertróficasqueloides y hernias umbilicales ${ }^{[9]}$.

El cáncer de páncreas es el décimo segundo cáncer más común en el mundo. En el Perú, según el Registro de Cáncer de Lima Metropolitana, la incidencia es de 3,9 por 100 mil habitantes y por lo menos $50 \%$ de este tipo de cáncer se detecta en fase avanzada (metastásico), y su tasa de sobrevida es de aproximadamente cinco años, por lo cual es importante hacer un diagnóstico temprano oportuno ${ }^{[10]}$.

La presentación clínica más frecuente de carcinoma de páncreas incluye síntomas como dolor en cuadrante abdominal superior o epigastrio, ictericia, náuseas o vómitos, diarrea y esteatorrea por insuficiencia hepática, cuando se trata de cáncer de cabeza de páncreas; mientras que, un dolor de espalda nuevo o que empeora podría ser indicativo de cáncer de cuerpo y cola. La clínica de esta enfermedad también incluye manifestaciones sistémicas como pérdida de peso rápida, anorexia y enfermedad tromboembólica ${ }^{[11,12]}$.

Las metástasis cutáneas de cáncer de páncreas son poco frecuentes $y$, cuando ocurren, son umbilicales. Hay reportes de metástasis en otras localizaciones como el cuello y cuero cabelludo. En la mayoría de los casos el tumor se origina en cuerpo y cola del páncreas, de la misma forma que en nuestro caso, a pesar que el $70 \%$ de los tumores de páncreas corresponden a adenocarcinoma de cabeza de páncreas ${ }^{[13]}$.

La presentación del nódulo de la hermana Mary Joseph puede ser, tal como el caso de nuestro paciente, la única manifestación previa a la aparición de síntomas de la neoplasia subyacente. Dicha presentación coincide con los reportes de Vallejo et al. ${ }^{[14]}$ 

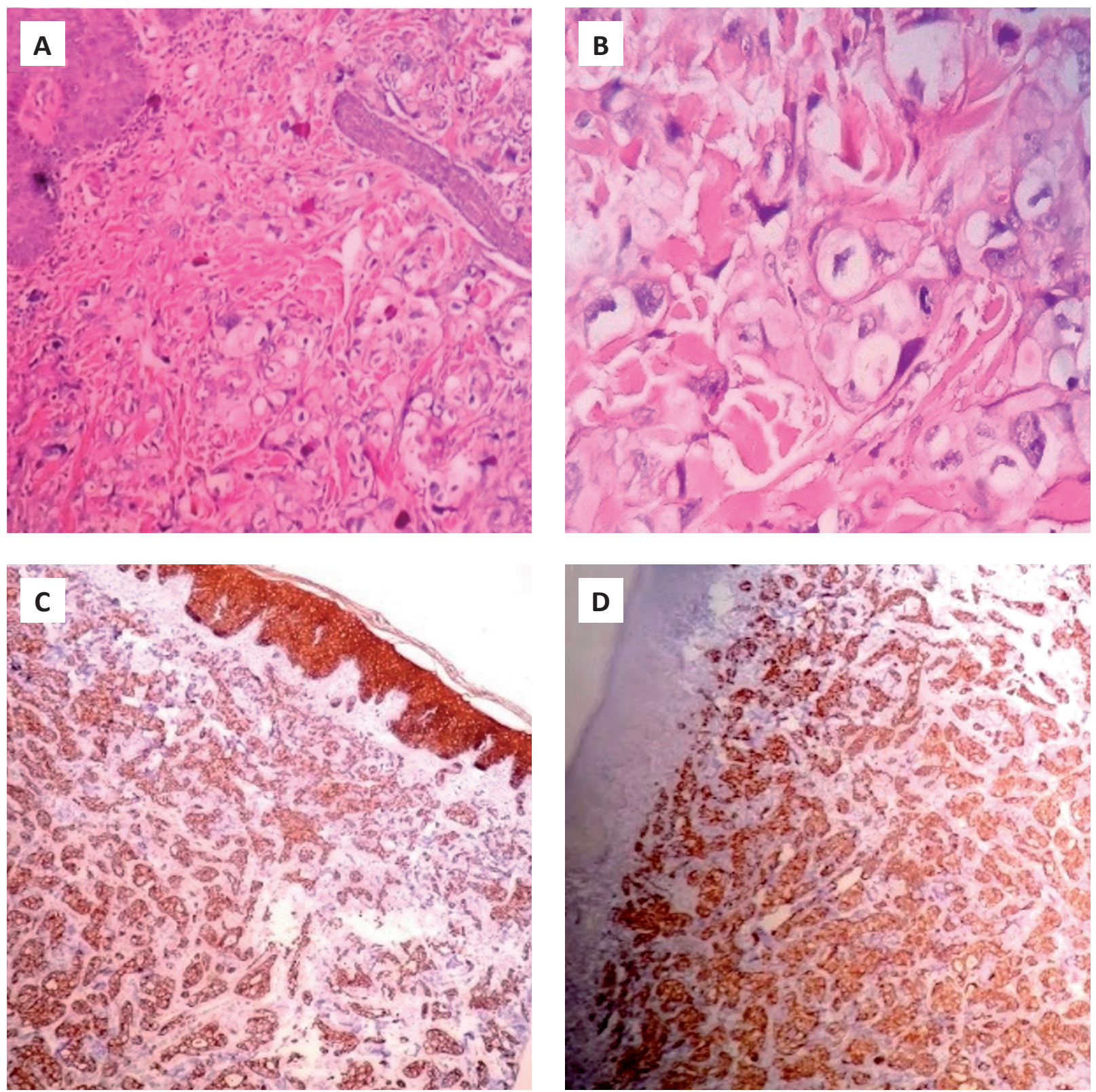

Figura 3. A) Se observa en dermis, múltiples células que se disponen en estructuras similares a ductos (tinción hematoxilina y eosina [H\&E], $10 \mathrm{X}$ ). B) A mayor aumento se aprecian mejores las estructuras ductales en dermis media con células atípicas (H\&E. $40 \mathrm{X}$ ). C) Células marcan positivas para panqueratina. D) Positivo para citokeratina 7.

y Lopez Corral, donde el nódulo umbilical también fue la única manifestación clínica del paciente, previa al diagnóstico de la neoplasia. En el caso de Vallejo et al., la lesión fue confundida inicialmente con una hernia inguinal, puesto que el paciente refería dolor y protrusión umbilical como síntomas principales.

En la tomografía computarizada se suele observar una imagen tumoral hiperdensa que capta el contraste, como en el reporte de Sharma et al., cuya manifestación clínica e imagen tomográfica fueron semejantes a las de nuestro paciente ${ }^{[15]}$.

La biopsia del nódulo es fundamental en el diagnóstico del origen del tumor ya que las características histológicas e inmunohistoquímicas nos permiten definir el origen de la neoplasia. El tipo histológico más frecuente es el adenocarcinoma ${ }^{[16]}$, como ocurrió en nuestro paciente. Así mismo, la muestra positiva para CK7 (que puede ser positiva en órganos de origen glandular como ovario, mama, páncreas, tiroides, etc.) y panqueratina (útil para neoplasias epiteliales entre ellas las de origen gastrointestinal) permitieron realizar el diagnóstico de nódulo metastásico de carcinoma de páncreas. En estos casos el marcador CK20 es de gran utilidad ${ }^{[17]}$; sin embargo, no pudo realizarse en nuestro paciente.

Entre los diagnósticos diferenciales pueden incluirse hernia umbilical, endometriosis cutánea, granuloma piógeno, cicatriz 
queloide, melanoma, carcinoma de células escamosas, entre otras $^{[18]}$.

Algunos autores proponen el tratamiento agresivo que combina la extirpación quirúrgica, quimioterapia y radioterapia; mientras que otros estudios plantean ofrecer solo tratamiento paliativo debido a que todos los pacientes suelen diagnosticarse en etapas avanzadas de la enfermedad ${ }^{[19,20]}$.

En nuestro paciente la supervivencia fue menor a lo reportado de 10 meses ${ }^{[21]}$, incluso a pesar de la quimioterapia descrita para el manejo de este caso ${ }^{[22]}$; no obstante, algunos estudios muestran que la supervivencia podría expandirse aproximadamente a 17,6 meses con quimioterapia ${ }^{[23]}$.

La limitación de nuestro trabajo se debe al hecho de ser reporte de un caso, siendo necesario realizar una mayor cantidad de observaciones o un estudio epidemiológico para tener mayor conocimiento de esta forma de presentación cutánea en nuestra literatura; sin embargo, consideramos importante su publicación por ser de infrecuente aparición, como única manifestación clínica inicial, que se ha observado solo en algunos reportes en la literatura actual.

En conclusión, la presentación de un nódulo umbilical con síntomas abdominales obliga a descartar no sólo neoplasia de tracto gastrointestinal sino de otros órganos principalmente intrabdominales, para así realizar un diagnóstico oportuno de una condición seria con grave impacto en la supervivencia del paciente.

Fuente de financiamiento: El autor declara no haber recibido ninguna financiación para la realización de este trabajo.

Declaración de conflicto de intereses: Los autores declaran no tener conflicto de intereses con la publicación de este artículo.

\section{REFERENCIAS BIBLIOGRÁFICAS}

1. Miranda A, Alves M, Lopes F. Sister Mary Joseph' nodule. Rev Clin Esp. 2014;214(4): e47.

2. Miller T, Ashworth J, Richards S. Sister Mary Joseph nodule. BMJ. 2015;351:h5224.

3. Espinel J, Pinedo E, Ojeda V. Sister Mary Joseph's nodule. Rev Esp Enferm Dig. 2016;108(2):97

4. Martínez L, Corell V. Metástasis cutáneas de neoplasias internas. Med Cutan Iber Lat Am. 2009;37(3):117-29.
5. Urbano F. Sister Joseph's nodule. Hospital Physician. 2001;37(5):3344.

6. Menzies S, Chotirmall S, Wilson G. Sister Mary Joseph nodule. BMJ Case Rep. 2015;2015. pii: bcr2014206808.

7. Sánchez Y, Estrada G. La imagen en la historia: revisión histórica y pictórica del nódulo de la hermana María José en cinco casos. Anales Radiol México. 2007;4:327-32.

8. Davar S, Hanna D. Sister Mary Joseph's nodule. J Cutan Med Surg. 2012;16(3):201-4.

9. Chirapongsathorn S. Sister Mary Joseph's nodule. Mayo Clinic Proceedings. 2014;90(2):310.

10. Rebaza S. Cáncer de páncreas. Rev Gastroenterol Peru. 2016;36(2):105-6.

11. Kanji ZS, Gallinger S. Diagnosis and management of pancreatic cancer. CMAJ. 2013;185(14):1219-26.

12. Seyfried TN, Huysentruyt LC. On the origin of cancer metastasis. Crit Rev Oncog. 2013;18(1-2):43-73.

13. Bai X.-L, Zhang Q, Masood W. Sister Mary Joseph's nodule as a first sign of pancreatic cancer. World J Gastroenterol. 2012;18(45):6686-9.

14. Vallejo C, Casamayor MC, Hakim S. Nódulo de la hermana María José como primera manifestación de cáncer pancreático. Rev Esp Enferm Dig. 2017;109(2):167-8.

15. Sharma A, Sharma V. Image Diagnosis: Sister Mary Joseph Nodule. Perm J. 2014;18(2):e132.

16. Akram M, Baig MA, Ali S. Sister Mary Joseph nodule, a forgotten nodule. J Ayub Med Coll Abbottabad. 2014;26(3):416-8.

17. Zhu S, Xichuan Y. Sister Mary Joseph's nodule as a diagnostic clue to metastatic colon carcinoma. J Clin Oncol. 2009;27(19):e1-2.

18. Vagnonii M. Metástasis umbilical: nódulo de la Hermana María José. Arch Argent Dermatol. 2014;64(4):165-7.

19. Talebi S, Chaudhari S. Sister Mary Joseph nodule. QJM. 2016;109(6):419-20.

20. Pereira W, Humaire C, Simon C. Sister Mary Joseph's nodule: a sign of internal malignancy. An Bras Dermatol. 2011;86(4):118-20.

21. Iavazzo C, Madhuri K, Essapen S. Sister Mary Joseph's nodule as a first manifestation of primary peritoneal cancer. Case Rep Obstet Gynecol. 2012;2012:467240.

22. Ducreux M, Cuhna AS, Caramella C, Hollebecque A, Burtin P, Goéré $D$, et al. Cancer of the pancreas: ESMO Clinical Practice Guidelines for diagnosis, treatment and follow-up. Ann Oncol. 2015;26 Suppl 5:v56-68.

23. Bdeiri K, Kamar FG. Cutaneous metastasis of pancreatic adenocarcinoma as a first clinical manifestation: a case report and review of the literature. Gastrointest Cancer Res. 2013;6(2):61-3. 